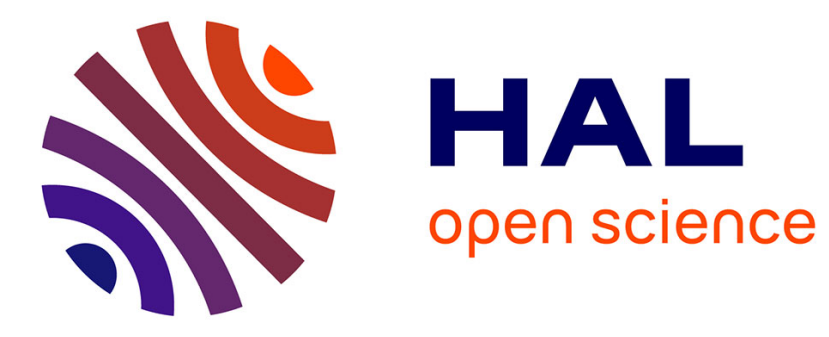

\title{
Polycrystalline Plasticity Under Small Strains
}

\author{
Fabrice Barbe, Samuel Forest, Georges Cailletaud
}

\section{To cite this version:}

Fabrice Barbe, Samuel Forest, Georges Cailletaud. Polycrystalline Plasticity Under Small Strains. Bouchaud E., Jeulin D., Prioul C., Roux S. (eds). Physical Aspects of Fracture. NATO Science Series (Series II: Mathematics, Physics and Chemistry), 32, Springer Netherlands, pp.191-206, 2001, 978-0-7923-7147-2. 10.1007/978-94-010-0656-9_15. hal-02327413

\section{HAL Id: hal-02327413 \\ https://hal.science/hal-02327413}

Submitted on 22 Oct 2019

HAL is a multi-disciplinary open access archive for the deposit and dissemination of scientific research documents, whether they are published or not. The documents may come from teaching and research institutions in France or abroad, or from public or private research centers.
L'archive ouverte pluridisciplinaire HAL, est destinée au dépôt et à la diffusion de documents scientifiques de niveau recherche, publiés ou non, émanant des établissements d'enseignement et de recherche français ou étrangers, des laboratoires publics ou privés. 


\title{
POLYCRYSTALLINE PLASTICITY UNDER SMALL STRAINS
}

Toward finer descriptions of microstructures

\author{
F. BARBE, S. FOREST, G. CAILLETAUD \\ Centre des Matériaux/UMR 7633, Ecole des Mines \\ de Paris/CNRS, BP87, F-91003 EVRY, France
}

\begin{abstract}
In the study of the mechanics of heterogeneous materials, FE method benefits from the rising of computing power. Within the framework of crystal plasticity, it is now possible to describe the intragranular behavior in realistic 3D polycrystals and to reach a local converged representation of fields. This gives new insight into the study of grain boundary effects. Here, attention is focused on the minimum mesh-refinement to get a valid response on each scale of the modeling. A comparison with a homogenization model is also made.
\end{abstract}

\section{Introduction}

After the pioneering work due to Taylor [1], crystal plasticity is a classical topic in the literature. A series of models have been elaborated in the past. Single crystal models came first, with a first type of models in the sixties, mainly applied to pure metals [2], and a second generation able also to represent more complex behaviors, for instance superalloys, in the eighties [3-5]. Transition rules from the macroscopic scale to the microscopic scale in the framework of simplified approaches, and the related homogenization theories, have been developed in the same time [6-9], allowing the user to account for strain or stress heterogeneities in a polycrystalline material element, with the assumption of uniform values in each phase. This last assumption is rather strong, since it does not account for the stress/strain redistribution into the grains, and cannot give any idea of important features like surface effect and grain boundary effect.

On the other hand, the present state of the computing power offers now the possibility to reach original results with FE methods, especially in the study of the mechanics of heterogeneous materials. The first studies have been performed by describing a polycrystal with uniform shape of grains -cubic or polyhedral- or even several grain orientations comprised in a single element. One of the issues successfully addressed with FE deals with the prediction of the mean behavior of polycrystals at large strains together with texture evolution [10-16]. Still, for large strains, neither the distribution of orientations inside the structure nor the shape of the grains have been proved to have a large importance for the purpose of texture evolution $[14,16]$. Another kind of problem addressed with FE is the description of plasticity inside grains of a multicrystal with realistic grain shapes [17-19]. Such studies can only rely on quasi-3D structures made of a small number of large grains since experimental determination of the real 3D morphology of a polycrystal 
is still a difficult task. Their constitutive models take into account the evolution of density of dislocations and they resort to a large number of elements per grain to describe the intragranular behavior and the effects of the grain boundaries. An intermediate problem lying between the case of idealized polycrystals and this of real multicrystalline structures consists in representing each grain of a polycrystal with Voronoï cells, either Voronoï cell elements [20] or several elements inside each grain $[21,22]$; but these works are usually restricted to $2 \mathrm{D}$ structures.

The purpose of this paper is to present the results obtained in the FE simulation of a 3D polycrystalline aggregate, with a realistic microstructure. Section 2 presents the crystallographic model which can be applied to represent single crystal behavior. An original transition rule, the $\beta$-model [23], is also briefly discussed, in relation with a simpler model [9], which will be used as a reference in our computations. Then, in Section 3, we present computations on a 3D polycrystal with grains having the shapes of Voronoï polyhedra, as was first proposed in [24] and then in $[25,26]$. In particular, in Section 3.2, a varying number of elements is used in order to determine how many full integration 20-node brick elements per grain are required, in average, to get a valid response on such or such a scale. The method to generate 3D microstructures is first recalled in Section 3.1.

\section{Model for the Single- and Polycrystal}

\subsection{Single Crystal}

It is assumed that slip is the predominant deformation mechanism and that Schmid's law is valid. The resolved shear stress can then be used as a critical variable to evaluate the inelastic flow. A viscoplastic framework is chosen, in order to avoid the problems related to the determination of the active slip systems in plastic models. A threshold is introduced both in positive and negative directions on each slip system : twelve octahedral slip systems will be used for FCC materials. Two variables are defined for each slip system $s, r^{\mathrm{s}}$ and $x^{\mathrm{s}}$, corresponding respectively to isotropic hardening (expansion of the elastic domain), and kinematic hardening (translation of the elastic domain [27]). A system will be active provided its resolved shear stress $\tau^{\mathrm{s}}$ is greater than $x^{\mathrm{s}}+r^{\mathrm{s}}$ or less than $x^{\mathrm{s}}-r^{\mathrm{s}}$ and the slip rate will be known as long as stress and the hardening variables are known. The state variables used to define the evolution of $r^{\mathrm{s}}$ and $x^{\mathrm{s}}$ are the accumulated slip $v^{\mathrm{s}}$ for isotropic hardening and the variable $\alpha^{\mathrm{s}}$ for kinematic hardening. Knowing the stress tensor $\sigma^{\mathrm{g}}$ applied to the grain $\mathrm{g}$, the resolved shear stress for system $s$ can be classically written according to (1), $\vec{n}^{\mathrm{s}}$ and $\vec{m}^{\mathrm{s}}$ being respectively, for the system $s$, the normal to the slip plane and the slip direction in this plane. The hardening variables $x^{\mathrm{s}}$ and $r^{\mathrm{s}}$ can then be expressed as a function of $\alpha^{\mathrm{s}}$ and $v^{\mathrm{s}}$ following (2), their actual values allowing then to compute the viscoplastic slip rate $\dot{\gamma}^{\mathrm{s}}$, the viscoplastic strain rate tensor $\underset{\dot{\varepsilon}^{g}}{\mathrm{~g}}(3)$, and the hardening rules ((4) and (5)). The present formulation gives a saturation of the hardening in both monotonic and cyclic loading, 


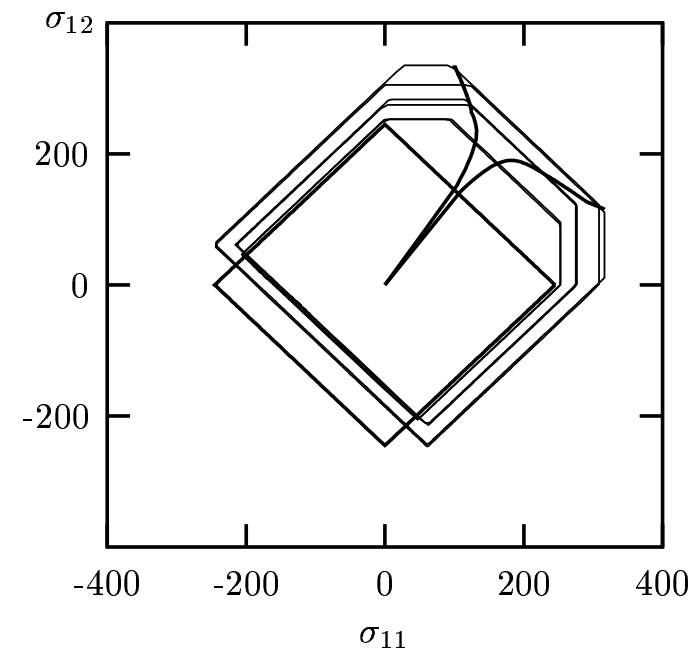

\begin{tabular}{|c|c||c|c|}
\hline \multicolumn{4}{|c|}{ Coefficients (MPa, s) } \\
\hline$\tau_{0}$ & 100 & $c$ & 10000 \\
$Q$ & 20 & $d$ & 200 \\
$b$ & 10 & $K$ & 20 \\
$h_{i j}$ & 1 & $n$ & 2 \\
\hline
\end{tabular}

Upper path : $\varepsilon_{12} / \varepsilon_{11}=0.525$

Lower path : $\varepsilon_{12} / \varepsilon_{11}=0.475$

Figure 1: Illustration of the bifurcation in the stress plane observed for prescribed similar strain paths in the case of a FCC single crystal

and takes into account the interactions between the slip systems, through matrix $h_{r s}$, as in [28]. Nine material-dependent coefficients are involved in the model $\left(E, \nu, K, n, c, d, R_{0}, Q, b\right)$.

$$
\begin{gathered}
\tau^{\mathrm{s}}=\sigma_{\sim}^{\mathrm{g}}:{\underset{\sim}{\mathrm{m}}}^{\mathrm{s}}=\frac{1}{2} \sigma^{\mathrm{g}}:\left(\vec{n}^{\mathrm{s}} \otimes \vec{m}^{\mathrm{s}}+\vec{m}^{\mathrm{s}} \otimes \vec{n}^{\mathrm{s}}\right) \\
x^{\mathrm{s}}=c \alpha^{\mathrm{s}} \quad ; r^{\mathrm{s}}=R_{0}+Q \sum_{r} h_{r s}\left\{1-e^{-b v^{\mathrm{r}}}\right\} \\
\dot{\gamma}^{\mathrm{s}}=\dot{v}^{\mathrm{s}} \operatorname{sign}\left(\tau^{\mathrm{s}}-x^{\mathrm{s}}\right) \quad ; \quad \dot{\sim}^{\mathrm{g}}=\sum_{s}{\underset{\sim}{m^{\mathrm{s}}} \dot{\gamma}^{\mathrm{s}}}^{\mathrm{s}}=\left\langle\frac{\left|\tau^{\mathrm{s}}-x^{\mathrm{s}}\right|-r^{\mathrm{s}}}{K}\right\rangle^{n} \quad \operatorname{with}\langle x\rangle=\operatorname{Max}(x, 0) \quad \text { and } \quad v^{\mathrm{s}}\left(t=t_{0}\right)=0 \\
\dot{\alpha}^{\mathrm{s}}=\dot{\gamma}^{\mathrm{s}}-d \alpha^{\mathrm{s}} \dot{v}^{\mathrm{s}} \quad \text { with } \quad \alpha^{\mathrm{s}}\left(t=t_{0}\right)=0
\end{gathered}
$$

Such a formulation $[4,29]$ is an extension of the classical crystallographic approach for single crystal modeling in plasticity or in viscoplasticity (see for instance $[1,2,30])$. It has been extensively used for single crystal modeling including Finite Element simulations [31,32]. For this type of approach, as illustrated in fig.1, the presence of corners in the yield surface may produce strong variations of the flow direction for a given loading. This fact can be important in aggregate computations, since complex tridimensional loading paths will be applied to each integration point. 


\subsection{Transition rule for the polycrystal}

In a polycrystalline aggregate, one phase may be characterized by its shape, size, crystallographic orientation, location with respect to the surface of the material, etc ... Most of the models usually specified for polycrystals made of equiaxial grains retain only the crystallographic orientation $[10,15,16]$, and put in the same crystallographic phase all the grains having the same orientation. Thus the alloy is considered as a $n$-phase material, each phase being defined by a set of Euler angles, and then the model is used to describe the mean behavior of all of them.

The relation (6) summarizes the results given by several models, according to the definition of $\alpha$, with a specific mention to $\alpha=2$ [33] representing uniform total strain, to $\alpha=1$ [6], corresponding to an elastic accommodation of the homogeneous medium, or to (7) involving the overall equivalent stress $\Sigma$ in uniaxial tension and the equivalent plastic strain $p$ deduced from the overall plastic strain tensor $\underset{\sim}{E^{p}}[9]$. This last model is the simplified expression extracted from the general self-consistent model due to Hill [7], valid for isotropic elasticity and radial loading paths.

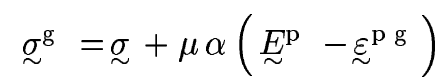

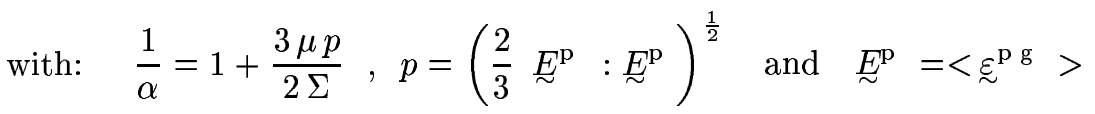

From a physical point of view, the previous rules simply show that a local plastic strain decreases the local stress, whereas the stress redistribution related to plastic accommodation tends to decrease for larger plastic strains. An alternative formulation, the " $\beta$-model", introducing a non linear accommodation, has also been proposed [23]. It can be calibrated from finite element computations, using either an inclusion embedded in a homogeneous medium [34] or a $3 \mathrm{D} \mathrm{FE}$ polycrystal [35]:

$$
\begin{gathered}
\sigma^{\mathrm{g}}=\underset{\sim}{\sigma}+\mu\left(\underset{\sim}{\beta}-{\underset{\sim}{\beta^{g}}}^{g}\right) \\
\text { with } \underset{\sim}{\beta}=\sum_{g} f^{g}{\underset{\sim}{\beta^{g}}}^{g}
\end{gathered}
$$

Various expressions can be used for the evolution of the variable $\beta^{g}$ in each grain [36]. The model can be used for any kind of loadings, especially cyclic loadings.

Since the loading path in the present paper is just a tension, the BerveillerZaoui's (BZ) evaluation of the self-consistent scheme will be considered as a reference for the polycrystal behavior. The following coefficients are then used:

- isotropic elastic behavior: $\quad E=196000 M P a, \nu=0.3$

- viscous effect in (4) and (5): $K=10 M P a . s^{1 / n}, \quad n=25$

- kinematic hardening in (2): $\quad c=1600 M P a, d=40$

- isotropic hardening in (2) and (3): $R_{0}=111 M P a, Q=35 M P a, b=7$ 

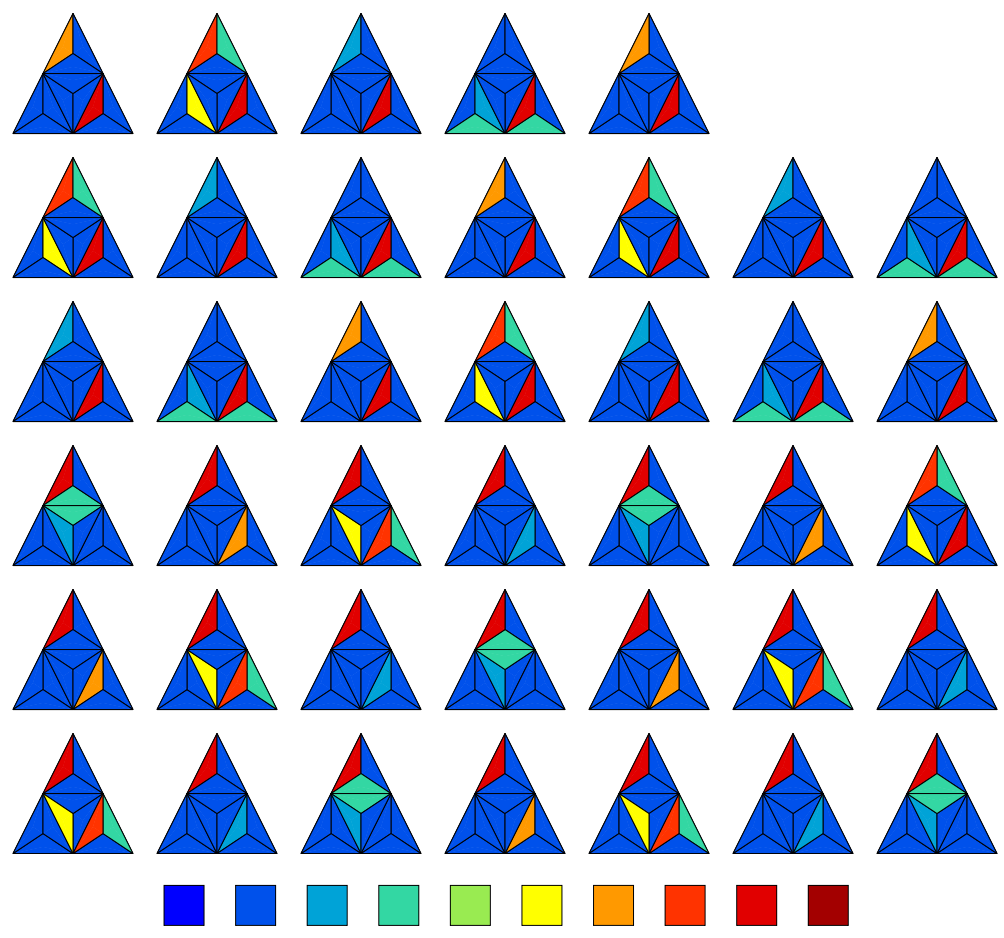

0

0.005

0.010

0.015

0.020

Figure 2: Values of the accumulated slip on each slip system for a $1 \%$ tension on a 40-grain aggregate with the BZ model

With such a kind of approach, one has an access to local quantities, like stresses or strains, but assuming that their values are uniform for each crystallographic phase. This is illustrated for example in fig.2, showing the amount of slip for each slip system in a FCC crystal presenting octahedral slip. In each "grain", conventionnally represented by an unfolded Thomson tetrahedron, the twelve slip systems are distributed on four large triangles figuring the slip planes, with 3 slip directions on each. One can observe that, even with a viscoplastic formulation, the number of active slip systems remains low, due to the presence of a threshold.

\section{Computing on cubic polycrystals}

As an example, we consider a single cubic polycrystal made of 200 grains. The original polycrystalline medium is not built from measures on real microstructures but generated in the form of a 3D voxel map representing Voronoï polyhedra (fig.3a). The procedure is explained below. Each grain in the FE structure is defined by a set of integration points rather than a set of elements. The elements 

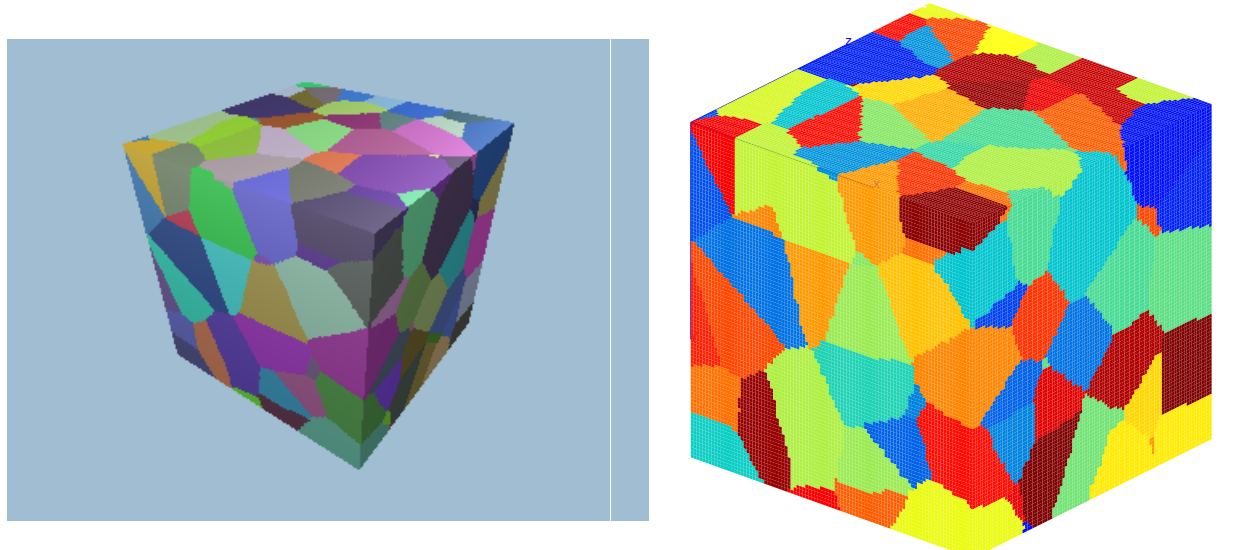

Figure 3: Aggregate made of 200 grains; (left) original map with $200^{3}$ voxels, (right) FE representation with $32^{3}$ 20-node-brick elements (884736 integration points)

are full-integration 20-node bricks -thus containing 27 integration points.

\subsection{Polycrystal generation}

Formally, Voronoï polyhedra are defined as zones of influence of a particular set of points, corresponding to their centers. Let $D \subset R^{3}$, and $E=\left\{A_{i}\right\}$ be a set of $N$ random points $P(x, y, z)$ corresponding to the centers of grains in the continuous domain $D$. If $d\left(P_{1}, P_{2}\right)$ is the euclidean distance between two points $P_{1}$ et $P_{2}$, the zone of influence of a point $A_{i}$ is defined in (10) by the set of points $P(x, y, z)$ with:

$$
i z\left(A_{i}\right)=\left\{P(x, y, z) \in D \quad \mid \quad d\left(P, A_{i}\right)<d\left(P, A_{j}\right) \quad \forall j \neq i\right\}
$$

In more physical terms, a point $P$ belongs to the zone of influence of germ $A_{i}$ if it is closer to $A_{i}$ than to any other germ. By construction, this zone of influence generates the Voronoï polyhedron centered in $A_{i}$. The set of zones of influence $\left\{i z\left(A_{i}\right)\right\}$ builds a random tesselation of the domain $D$ into $N$ classes, every $A_{i}$ being the germ of one class (fig.3a).

A specific procedure was developed to build Voronoï polyhedra inside a discrete domain, made of a 3D voxel map [37]. Each voxel is assigned the number (or label) of the polyhedron to which it belongs. Then, each integration point of the mesh is assigned the number -label- of the voxel at the same location in space. In the case proposed here (fig.3), there are $200^{3}$ voxels vs $96^{3}$ integration points in the most refined mesh.

\subsection{Computations and effects of mesh refinement}

Now we present a comparison of the results of the same simple tensile test on the aggregate of fig. 3 computed with varying number of elements: at the macroscopic scale, at the scale of the mean response per grain and at the intragranular scale. 


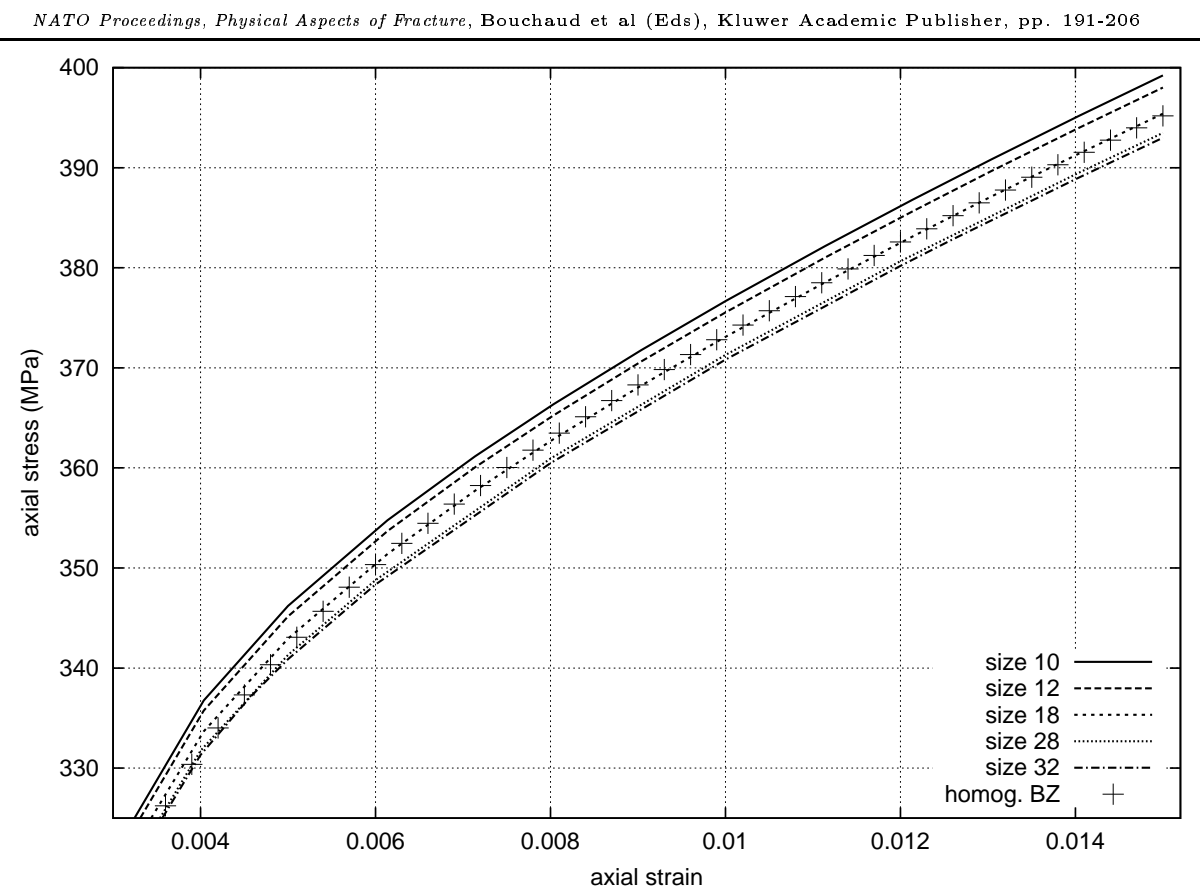

Figure 4: Mean axial stress-strain curves with five different sizes of mesh and with homogenization model

\section{Macroscopic scale}

Each structure, made respectively of $10^{3}, 12^{3}, 18^{3}, 32^{3}$ elements, is loaded to $E_{z z}=1.5 \%$ with homogeneous strain boundary conditions. The components of the loading strain tensor result from a preliminary homogenization simulation where the lateral stresses $\sigma_{x x}$ and $\sigma_{y y}$ were imposed to remain null. On fig.4, the mean stress-strain curve of the 200-grain-polycrystal is well reproduced whatever our conditions of modeling are: would it be homogenization or FE modeling with $10^{3}$ elements, the response of the $32^{3}$-mesh is respected with less than $1.5 \%$ of deviation in stress. The curves also illustrate the effect of the stiffness of the mesh: for a lower number of elements, the response becomes harder.

One may think that, imposing a same strain tensor to all the nodes of the contours, the structures have less freedom to behave on their own than in the case where only axial displacements are imposed to top and bottom faces of the cubes. This latter case with four free lateral faces have been presented in [26], for the same material and the same aggregate; in that case, the mean stress response was $7 \%$ lower than that with homogeneous strain boundary conditions. Considering the stress-strain curves of the grains, it has been shown that freeing lateral faces tends to promote the dispersion in strain whereas imposing homogeneous boundary conditions rises the dispersion in stress. So whether the mean stress-strain curves of fig. 4 would be more dispersed with free faces, or not, cannot be guessed without further investigation. 


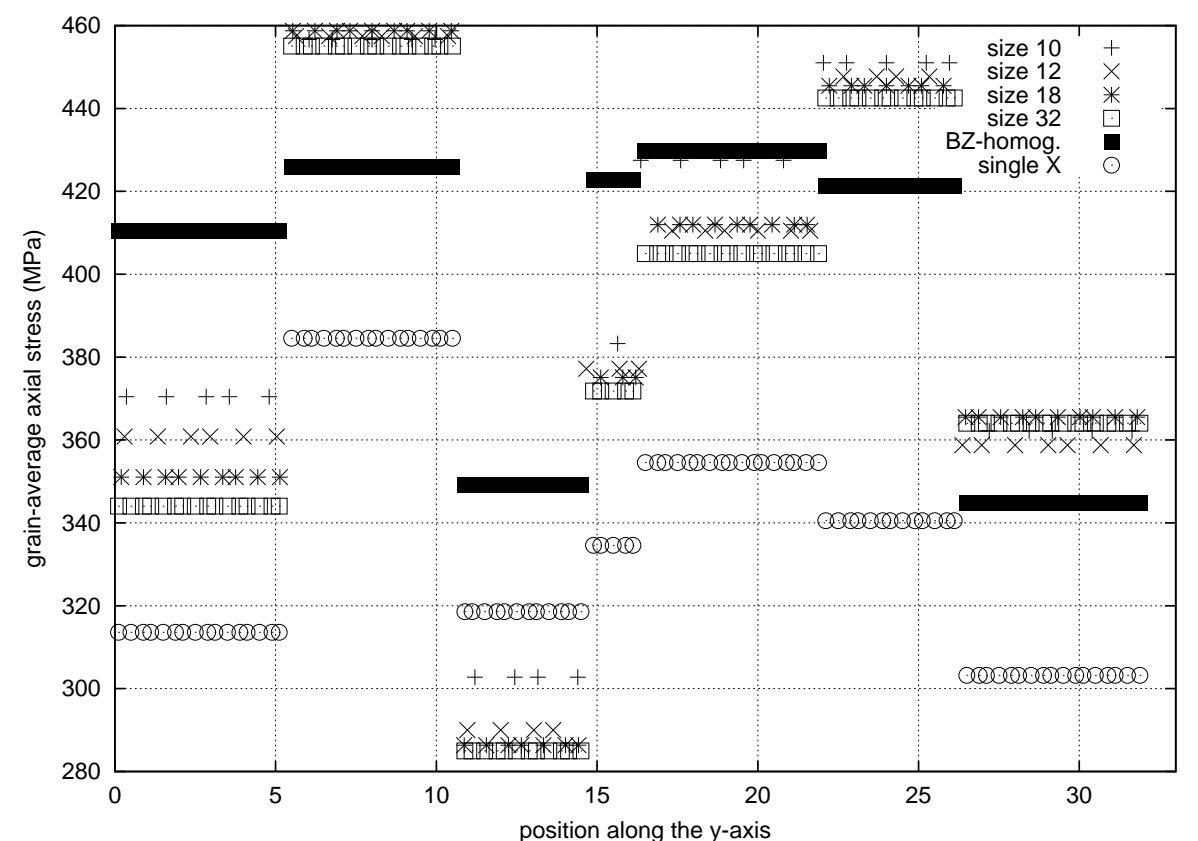

Figure 5: Mean axial stress in grains located along a line crossing the aggregate: mean stress per grain from four different sizes of mesh $\left(10^{3}, 12^{3}, 18^{3}, 32^{3}\right.$ elements), stress from the homogenization model, stress in the corresponding single crystals

\section{Mean grain scale}

The average response for each grain can be computed, and compared to the results of the homogenization (BZ) model. It has been made for this 200-grain aggregate in [26], for the same material. It appeared that BZ model underestimates the scatter in stress and strain between the different grains. This is due to the absence of local grain-to-grain interaction for the models with uniform stress/strain fields. Nevertheless, as long as 200 grains are considered on the same plot of stress-strain curves, it is difficult to identify an FE grain and its homogenization correspondent. So here we chose to show a comparison on grains located along a line of integration points crossing the structure (fig.5). This line is parallel to the lateral direction $y$ and is located in the middle of the structure. Since we deal with discretized domains and since refinement varies from $10^{3}$ elements to $32^{3}$, the location of the line varies slightly from a mesh to another. For the $10^{3}$-mesh it has the coordinates $x=z=14.40$, for $12^{3} x=z=14.42$, for $18^{3} x=z=14.42$ and for $32^{3} x=z=14.50$. These coordinates have been brought on the scale of the $32^{3}$-mesh (32 elements of size 1 in each direction, $0<x, y, z<32$ ). The volume of each grain along the line, divided by the average volume of a grain, gives respectively (from the left to the right): $1.28,0.96,1.42,1.26,0.76,2.02$, 1.87. Note that the number of points per grain appearing along the line is not related to the volume of the grain, since the line may pass through the middle of a grain or near its boundary. As a matter of fact, the $5^{t h}$ grain has the largest 
number of points intercepted by the line and is also the smallest grain.

Fig.5 shows the axial stress obtained from $4 \mathrm{FE}$ computations (10, 12, 18 and 32 elements along each edge of the cube), together with the self-consistent (BZ) response, and the response of the single crystal having the same orientation. This last value allows us to check if a given grain behaves simply according to its orientation or is strongly influenced by its neighborhood. Generally speaking, all the grains appear stronger in the polycrystal than as single crystals. This is due to the development of internal stresses. This effect can be classicaly observed, even for the self-consistent approach. Nevertheless, this fact is not necessary true for the FE computations, since stresses can be higher or smaller than stresses of the corresponding single crystals. The difference are even more pronounced $(>10 \%)$ in three of the grains (from the left, $1^{s t}, 3^{r d}$ and $4^{t h}$ ). As $1^{\text {st }}$ grain can be thought to be strongly influenced by the loading conditions in $\mathrm{FE}$ computations, $3^{\text {rd }}$ and $4^{\text {th }}$ cannot. Moreover, these grains have volumes higher than the average volume of a grain. So neither the volume nor the location in the structure are responsible for the difference between $\mathrm{BZ}$ and FE. The actual neighborhood of a grain can then be said to influence the mean stress response of a grain by more than $10 \%$ in stress, which may result in more than $60 \%$ in axial strain.

As the number of elements is increased, the accuracy of the mean response per grain is acceptable for all of the 200 grains with a minimum of $18^{3}$ elements. The $12^{3}$-mesh could also be considered as acceptable except that one can observe a difference in $1^{\text {st }}$ grain: there, the loading conditions obviously appear to influence the behavior. On the other hand, any kind of decrease of the elements number below $12^{3}$ produces non acceptable variations of the results. For instance, the mean behavior per grain provided by the $10^{3}$-mesh really departs from those of the other computations, especially in $3^{\text {rd }}$ and $5^{\text {th }}$ grains. $3^{\text {rd }}$ grain, though big its volume, is the only grain where the stress is lower than the one of the corresponding single crystal, which means that it is greatly influenced by its neighboring grains. $5^{\text {th }}$ grain is the smallest along the line. So it is not surprising to get the largest differences in these grains. Finally, as the discretization of the $12^{3}$-mesh corresponds to about 8 quadratic elements per grain, we will consider that $2 \times 2 \times 2$ quadratic elements and $6 \times 6 \times 6$ Gauss points are the critical numbers in a grain to get a decent estimation of its average stress.

\section{Intragranular scale}

As a first step toward the study of the local effect of mesh refinement, we propose to compare the local responses along the line used in the previous analysis, obtained from three sizes of mesh: $12^{3}, 18^{3}$ and $32^{3}$ elements (fig.6). The volume of each grain has already been defined and the number of integration points on each intersection with a grain can be counted from fig.5.

A von Mises stress plot is considered in fig.6a, and the cumulated slip on all the active slip systems in fig.6b (it is defined as the sum of the slips over the systems, $\left.\sum_{s}\left|\gamma^{\mathrm{s}}\right|\right)$. Surprisingly, the von Mises stress profiles are very similar for the three reported mesh sizes. The $12^{3}$-mesh nevertheless fails at reproducing the peak values in the $1^{\text {st }}$ and $5^{\text {th }}$ grains. On the other hand, for the case of the amount of 

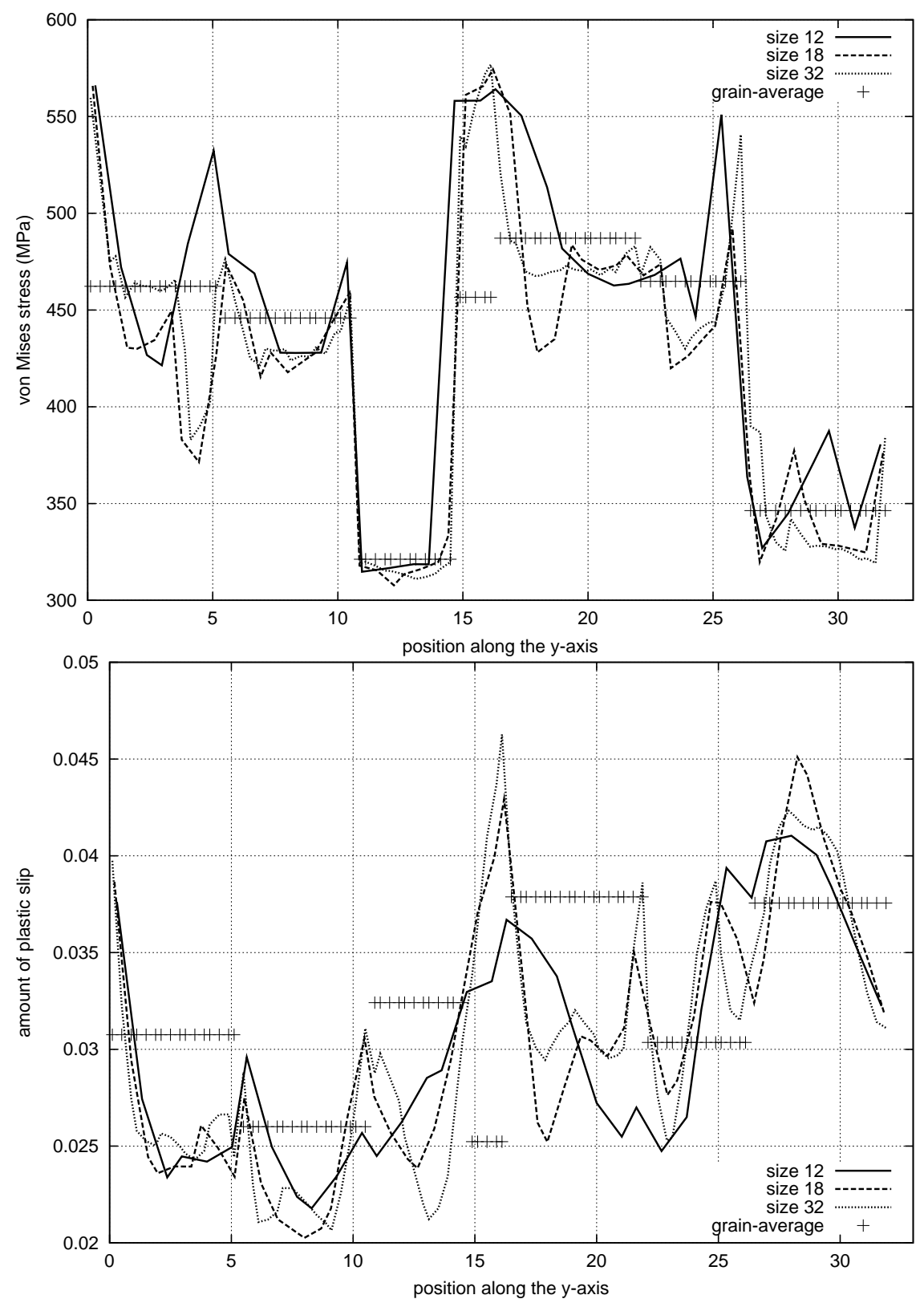

Figure 6: Plots along a line crossing the structure computed with three sizes of mesh $\left(12^{3}, 18^{3}, 32^{3}\right.$ elements): von Mises stress (upper), amount of plastic slip (lower). The mean response per grain obtained with $32^{3}$ elements is also figured 
plastic slip the lack of discretization becomes obvious: in $1^{s t}, 5^{\text {th }}$ and $6^{\text {th }}$ grains, it simply forgets or considerably attenuates peaks figured in $18^{3}$ and $32^{3}$-meshes. By 'peak' we mean an abrupt change in the direction of evolution, which could result in smoothing or hardening of the corresponding region. Such a region may then become a critical place where a crack initiation can preferentially occur under fatigue loading or a region of localized deformation resulting in slip bands. That is why with the refinement of the $12^{3}$-mesh, i.e. with about $2 \times 2 \times 2$ elements per grain, one cannot expect a correct representation of gradients inside grains. Fig.7a,c, showing the distribution of von Mises stress on the slices containing the lines of fig.6, allows to observe the poor intragranular resolution of the $12^{3}$-mesh.

Using a $18^{3}$-mesh, a good approximation of the local behavior is obtained, since nearly all the peaks are reproduced. The slight differences, in the amounts reached by the peaks or in the positions of the peaks cannot be said to come from a lack of discretization or from the difference in the location of the line of integration points. However, the comparison would probably not remain so good for any kind of line. This is demonstrated by fig.7 which show respectively the von Mises equivalent on a $12^{3}$-mesh (a), on a $18^{3}$-mesh (b), on a $32^{3}$-mesh (c), then the grain map (d), and finally the amount of plastic slip for the $18^{3}$-mesh (e) and for the $32^{3}$-mesh (f). The von Mises stress is well predicted only far from the grain boundaries since it is rather uniform inside each grain. But it is not the case for the amount of plastic slip: there are some grains where high-slip-activity-structures form near grain boundaries and where the rest of the grain remains unaffected by slip activity. Such features are strongly dependent on the resolution of the grain boundary: if the $18^{3}$-mesh only is considered, one may observe high-slip-activity-regions spreading over grain boundaries. But with a higher resolution, these structures appear to be disconnected at the grain boundaries, thus illustrating the fact that the slip activity is mainly due to the gradients of stress at the grain boundaries and not related to a kind of propagation of slip across boundaries.

Hence, having about $3 \times 3 \times 3$ elements per grain (case of $18^{3}$-mesh) may lead to a first good estimation of the gradients of the fields inside grains. Yet, for a systematic treatment aiming at describing the effect of grain boundaries (e.g. plotting field variables $v s$ the distance to the grain boundary and averaging over the grains of the aggregate [38]), the uncertainty resulting from the fact that grain boundaries pass inside elements instead of between elements (so-called multiphase elements) could alterate the observations made in the vicinity of the boundaries. Such a systematic treatment requires to have at least $4 \times 4 \times 4$ elements per grain so that one is assured that there are $2 \times 2 \times 2$ single-phase elements inside each grain, i.e. enough elements unaffected by the numerical construction of grain boundaries.

\section{Conclusion}

Comparisons of computations with a varying number of elements have been performed. For each scale of interest -macroscopic, mean grain scale, intragranular- a minimum number of elements per grain has been defined. Note that the elements 


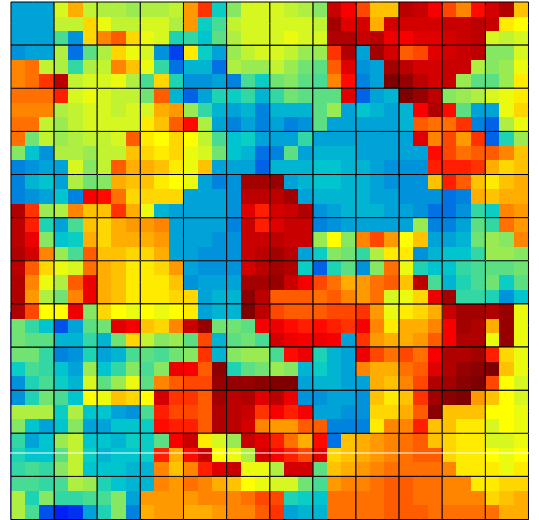

(a)

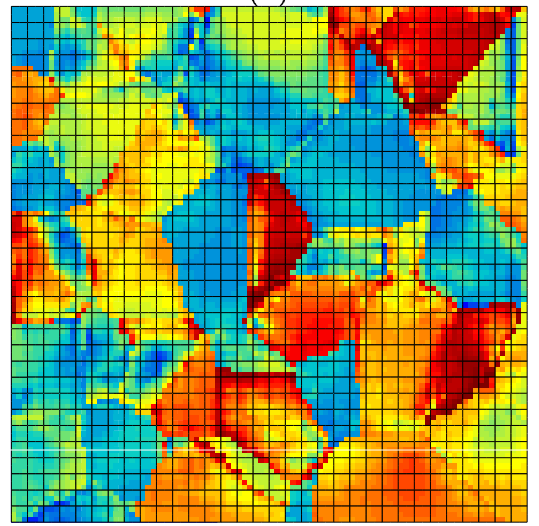

(c)

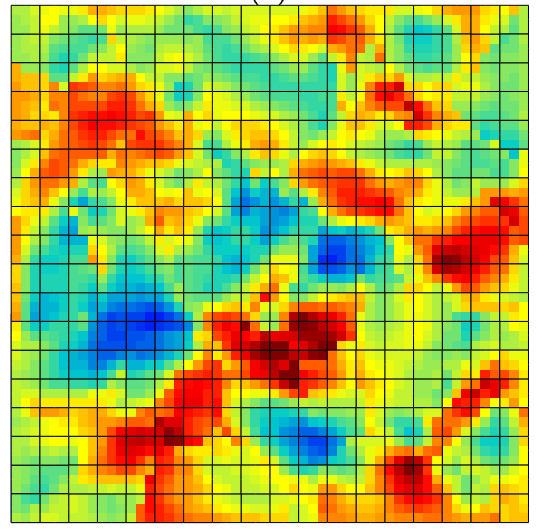

(e)

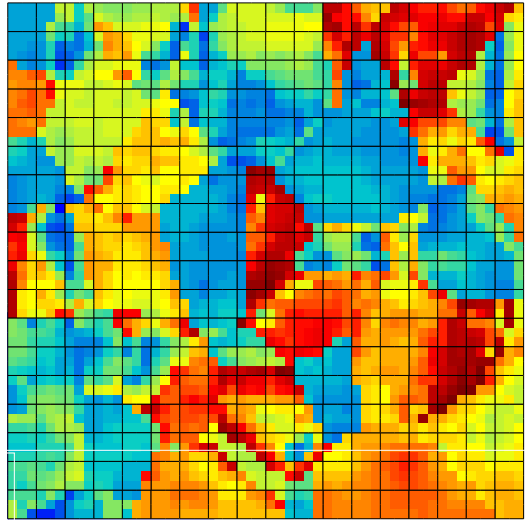

(b)

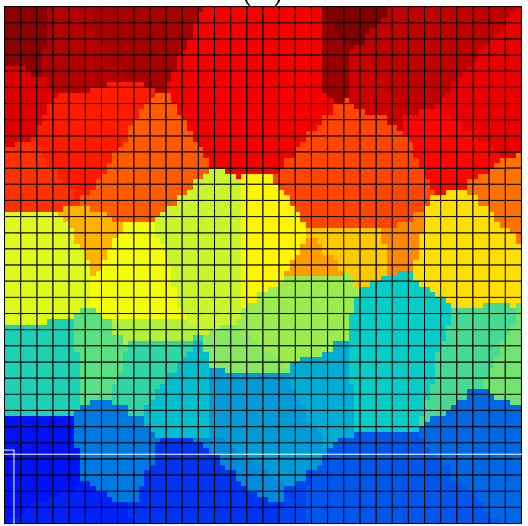

(d)

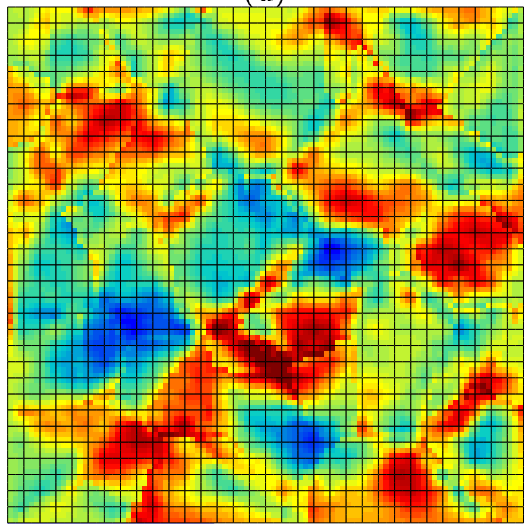

(f)

Figure 7: Contour plots on slices of integration points containing the lines investigated previously: (a) von Mises stress in $12^{3}$-mesh $(x=14.66)$, (b) von Mises stress in $18^{3}$-mesh $(x=14.42)$, (c) von Mises stress in $32^{3}$-mesh $(x=14.50)$, (d) grain map in $32^{3}$-mesh, (e) amount of plastic slip in $18^{3}$-mesh, (f) amount of plastic slip in $32^{3}$-mesh 
contain 27 integration points. So if 8-integration-points elements were to be used, our results could be useful as long as one adapts them by counting integration points rather than elements.

By combining those full-integration elements to the description of grains with sets of integration points (instead of sets of elements), one obtains an improved resolution of the grain boundaries. Analyzing the intragranular stress-strain curves of each integration point inside a grain, it has been found that the tremendous dispersion of the curves were not due to the multiphase elements. Further, studied as a function of the distance to the grain boundary, the variables featured a continuous spreading from the center to the grain boundary, without any visible step of variation in multiphase elements [38]. So the use of multiphase elements seems to be adequate.

The computations of 3D polycrystals require a huge amount of degrees of freedom but their use is not limited to the study of classical crystal plasticity. A direct application of such computations has been proposed recently: the calibration of a homogenization model with the mean behavior of an $\mathrm{FE}$ aggregate [35]. The method presented here has also been extended to size-sensitive crystal plasticity [39].

\section{References}

[1] G.I. Taylor. Plastic Strain in Metals. J. Inst. Metals, 62:307-324, 1938.

[2] Jean Mandel. Une généralisation de la théorie de la plasticité de W.T. Koiter. Int J Sol Struct, 1:273-295, 1965.

[3] E. Jordan and K.P. Walker. Biaxial Constitutive Modeling and testing of Single Crystals at Elevated Temperature. In Conference on Multiaxial Fatigue, Sheffield (UK), 1984.

[4] Laurent Méric, Philippe Poubanne, and Georges Cailletaud. Single Crystal Modeling for Structural Calculations. Part 1: Model Presentation. J Engng Mat Technol, 113:162-170, 1991.

[5] E.P. Busso and F.A. McClintock. A dislocation mechanics-based crystallographic model of a b2-type intermetallic alloy. Int J Plasticity, 12:1-28, 1996.

[6] E. Kröner. Zur plastischen Verformung des Vielkristalls. Acta Metall, 9:155$161,1961$.

[7] R. Hill. Continuum Micro-Mechanisms of Elastoplastic Polycrystals. J Mech Phys Sol, 13:89-101, 1965.

[8] J.W. Hutchinson. Elastic-Plastic Behaviour of Polycrystalline Metals and Composites. Proc R Soc London, A319:247-272, 1966.

[9] M. Berveiller and A. Zaoui. An Extension of the Self-Consistent Scheme to Plastically Flowing Polycrystal. J Mech Phys Sol, 26:325-344, 1979. 
[10] A.J. Beaudoin, K.K. Mathur, P.R. Dawson, and G.C. Johnson. Threedimensional deformation process simulation with explicit use of polycrystal plasticity models. Int J Plasticity, 9:833-860, 1993.

[11] R. Becker and S. Panchanadeeswaran. Effects of grain interactions on deformation and local texture in polycrystals. Acta metall mater, 43(7):2701-2719, 1995 .

[12] H. Takahashi, H. Motohashi, M. Tokuda, and T. Abe. Elastic-plastic finite element polycrystal model. Int J Plasticity, 10(1):63-80, 1994.

[13] Gorti B. Sarma and Paul R. Dawson. Texture predictions using a polycrystal plasticity model incorporating neighbor interactions. Int J Plasticity, 12(8):1023-1054, 1996.

[14] Venugopal Bachu and Surya R. Kalidindi. On the accuracy of the predictions of texture evolution by the finite element technique for fcc polycrystals. Mat Sc Engng, A257:108-117, 1998.

[15] A. Staroselski and L. Anand. Inelastic deformation of polycrystalline face centered cubic materials by slip and twinning. J Mech Phys Sol, 46(4):671696, 1998.

[16] D.P. Mika and P.R. Dawson. Polycrystal plasticity modeling of intracrystalline boundary textures. Acta mater, 47(4):1355-1369, 1999.

[17] C. Teodosiu, J.L. Raphanel, and L. Tabourot. Finite element simulation of the large elastoplastic deformation of multicrystals. In C. Teodosiu, J.L. Raphanel, and F. Sidoroff, editors, Proc. of the int. seminar Mecamat'91: Large Plastic Deformations, Fundamental Aspects and Applications to Metal Forming, Fontainebleau, France:153-168, August, 7-9 1991.

[18] Jörn Harder. A crystallographic model for the study of local deformation processes in polycrystals. Int J Plasticity, 15(6):605-624, 1999.

[19] F. Delaire, J.L. Raphanel, and C. Rey. Plastic heterogeneities of a copper multicrystal deformed in uniaxial tension: experimental study and finite element simulations. Acta mater, 48:1075-1087, 2000.

[20] Somnath Ghosh and Suresh Moorthy. Elastic-plastic analysis of arbitrary heterogeneous materials with the Voronoi Cell finite element method. Comput Meth Appl Mech Engng, 121:373-409, 1995.

[21] Osamu Watanabe, Hussein M. Zbib, and Eiji Takenoushi. Crystal plasticity: micro-shear banding in polycrystals using Voronoï tessalation. Int J Plasticity, 14(8):771-788, 1998. 
[22] E. Nakamachi, K. Hiraiwa, H. Morimoto, and M. Harimoto. Elastic/crystalline viscoplastic finite element analyses of single- and poly-crystal sheet deformations and their experimental verification. Int $J$ Plasticity, 16:1419-1441, 2000.

[23] G. Cailletaud and P. Pilvin. Utilisation de modèles polycristallins pour le calcul par éléments finis. Revue Européenne des Éléments Finis, 3(4):515$541,1994$.

[24] S. Quilici and G. Cailletaud. F.E. simulation of macro-, meso- and microscales in polycrystalline plasticity. Comput Mat Sc, 16(1-4):383-390, 1999.

[25] F. Barbe, L. Decker, D. Jeulin, and G. Cailletaud. Intergranular and intragranular behavior of polycrystalline aggregates. Part 1: F.E. model. Int $J$ Plasticity, 17:513-536, 2001.

[26] F. Barbe, S. Forest, and G. Cailletaud. Intergranular and intragranular behavior of polycrystalline aggregates. Part 2: results. Int J Plasticity, 17:537-563, 2001.

[27] Jean-Louis Chaboche. Constitutive Equations for Cyclic Plasticity and Cyclic Viscoplasticity. Int J Plasticity, 5:247-302, 1989.

[28] U.F. Kocks and T.J. Brown. Latent Hardening in Aluminium. Acta Metall, 14:87-98, 1966.

[29] Georges Cailletaud. Une approche micromécanique phénoménologique du comportement inélastique des métaux. PhD thesis, Université Pierre et Marie Curie, Paris 6, 1987.

[30] Robert J. Asaro. Crystal Plasticity. J. Appl. Mech., 50:921-934, 1983.

[31] L. Méric and G. Cailletaud. Single Crystal Modeling for Structural Calculations. Part 2: Finite Element Implementation. J Engng Mat Technol, 113:171-182, 1991.

[32] J. Besson, R. Leriche, R. Foerch, and G. Cailletaud. Object-Oriented Programming Applied to the Finite Element Method. Part II. Application to Material Behaviors. Revue Européenne des Éléments Finis, 7(5):567-588, 1998.

[33] T.H. Lin. Analysis of Elastic and Plastic Strains of Face Centered Cubic Crystal. J Mech Phys Sol, 5:143-149, 1957.

[34] Philippe Pilvin. The Contribution of Micromechanical Approaches to the Modelling of Inelastic Behaviour of Polycrystals. In André Pineau, Georges Cailletaud, and Trevor Lindley, editors, Fourth Int. Conf. on Biaxial/multiaxial Fatigue and Design, pages 3-19, London, 1996. ESIS 21, Mechanical Engineering Publications. 
[35] F. Barbe, R. Parisot, S. Forest, and G. Cailletaud. Calibrating a homogenization polycrystal model from large scale $\mathrm{FE}$ computations of polycrystalline aggregates. to appear in J Phys IV, 2001.

[36] J. Besson, G. Cailletaud, J.L. Chaboche, and S. Forest. Mécanique des matériaux inélastiques. to appear, Hermès, 2001.

[37] L. Decker and D. Jeulin. Simulation 3D de matériaux aléatoires polycristallins. Revue de Métallurgie, CIT/Science et Génie des Matériaux, February:271-275, 2000.

[38] Fabrice Barbe. Etude numérique de la plasticité d'agrégats polycristallins. $\mathrm{PhD}$ thesis, Ecole Nationale Supérieure des Mines de Paris, décembre 2000.

[39] S. Forest, F. Barbe, and G. Cailletaud. Cosserat modelling of size effects in crystals. Int $J$ Sol Struct, 37(46-47):7105-7126, 2000. 